Mapping multiplicative to additive noise

This content has been downloaded from IOPscience. Please scroll down to see the full text.

2014 J. Phys. A: Math. Theor. 47195001

(http://iopscience.iop.org/1751-8121/47/19/195001)

View the table of contents for this issue, or go to the journal homepage for more

Download details:

IP Address: 129.215.5.251

This content was downloaded on 05/06/2014 at 10:32

Please note that terms and conditions apply. 


\title{
Mapping multiplicative to additive noise
}

\author{
Katy J Rubin, Gunnar Pruessner and Grigorios A Pavliotis
}

Department of Mathematics, Imperial College London, 180 Queen's Gate, London SW7 2BZ, UK

E-mail: gunnar.pruessner@physics.org

Received 3 January 2014, revised 19 March 2014

Accepted for publication 2 April 2014

Published 23 April 2014

\begin{abstract}
The Langevin formulation of a number of well-known stochastic processes involves multiplicative noise. In this work we present a systematic mapping of a process with multiplicative noise to a related process with additive noise, which may often be easier to analyse. The mapping is easily understood in the example of the branching process. In a second example we study the random neighbour (or infinite range) contact process which is mapped to an Ornstein-Uhlenbeck process with absorbing wall. The present work might shed some light on absorbing state phase transitions in general, such as the role of conditional expectation values and finite size scaling, and elucidate the meaning of the noise amplitude. While we focus on the physical interpretation of the mapping, we also provide a mathematical derivation.
\end{abstract}

Keywords: multiplicative noise, phase transitions, non-equilibrium PACS numbers: 74.40.Gh, 68.35.rh

\section{Introduction}

In many stochastic processes, activity cannot recover once it has ceased. This feature is at the centre of absorbing state phase transitions [1] which in turn makes a significant part of the wider field of non-equilibrium phase transitions [2]. In an equation of motion of a single degree of freedom $\psi(g)$ as a function of time $g$, the feature enters as a multiplicative noise, such as

$$
\partial_{g} \psi(g)=f(\psi(g))+\sqrt{\psi(g)} \eta(g),
$$

where $f(\psi)$ is a generic function with the property $f(0)=0$ and $\eta(g)$ is a noise process, to be specified below. Crucially, if $\psi$ vanishes at any time $g^{*}$ it will remain 0 for all future times. This is exactly the feature expected in an absorbing state phase transition and, closely related, in Reggeon field theory [1, 3].

In this paper we will consider the case where $\eta(g)$ represents standard white noise and we will consider the Itō interpretation of the stochastic term in (1). This equation can be 
solved either numerically (or even analytically in special cases), or the corresponding FokkerPlanck equation that governs the evolution of the transition probability density can be studied. In the following, we will trace the origin of the multiplicative noise which will provide an alternative formulation of the process with additive noise; as an added benefit, nonlinearities may simplify significantly, making a direct solution of the stochastic differential equation (SDE) more feasible.

It should be emphasized that a SDE of the form (1), which we rewrite here for a multiplicative noise that is an arbitrary function of $\psi$

$$
\mathrm{d} \psi(g)=f(\psi(g)) \mathrm{d} t+\sqrt{\sigma(\psi(g))} \mathrm{d} W(g),
$$

where $W(g)$ denotes standard one-dimensional Brownian motion, does not provide us with a complete description of the dynamics, since noise in this equation (or, equivalently, the stochastic integral when writing it as an integral equation) can be interpreted in different ways, including the well known Itō and Stratonovich interpretations [4]. This is a modelling issue and it has to be addressed separately. The Wong-Zakai theorem [5] suggests that when thinking of white noise as an idealization of a noise process with a non-zero correlation time, then the noise in (2) should be interpreted in the Stratonovich sense. On the other hand, it is by now well known that for stochastic systems with more than one fast time scale (one being the correlation time of the approximation to white noise, the other being, e.g. a timescale measuring the inertia of the system), in the limit as these timescales tending to zero, we obtain a SDE that can be of Itō or Stratonovich type, or neither [6, 7]. Making the physically correct choice of the type of noise in (2) is crucial, since different interpretations of the noise lead to SDEs with qualitatively different features. A standard example is geometric Brownian motion: the long time behaviour of solutions to this equation (for fixed values of the parameters in the SDE) can be different for the Itō and Stratonovich SDEs [8]. In this paper we will choose the Itō interpretation of noise. It is well known that it is possible to switch between different interpretations of the noise by adding an appropriate drift (which, of course, changes generally the qualitative properties of solutions to the SDE).

SDEs with multiplicative noise exhibit a very rich dynamical behaviour including intermittency and noise induced transitions [9]. On the other hand, state-dependent noise leads to analytical, numerical and even statistical difficulties, i.e. it is more difficult to estimate statedependent noise from observations as opposed to estimating a constant diffusion coefficient; see, e.g. [10]. It is natural, therefore, to ask whether it is possible to find an appropriate transformation that maps SDEs with multiplicative noise to SDEs with additive noise. This is possible in one dimension: an application of Itō's formula to the function (assuming, of course, that this function exists)

$$
h(x)=\int^{x} \frac{1}{\sqrt{\sigma(\psi)}} \mathrm{d} \psi,
$$

enables us to transform (2) into an SDE with additive noise for the new process

$$
z(g)=h(\psi(g)),
$$

see, e.g. [4]. This transformation can also be performed at the level of the corresponding Fokker-Planck equation, see e.g. [11]. Such a transformation mapping multiplicative to additive noise does not generally exist in dimensions greater than one, unless the diffusion matrix satisfies appropriate compatibility conditions [10].

In this paper, we adopt a different approach: we introduce a new clock, so that, when measuring time with respect to this new time scale, noise becomes additive. Clearly, in order for this to be possible, the transformation to the new time must involve the actual solution of the SDE. The theoretical basis for this random time change is the Dambis-Dubins-Schwarz 


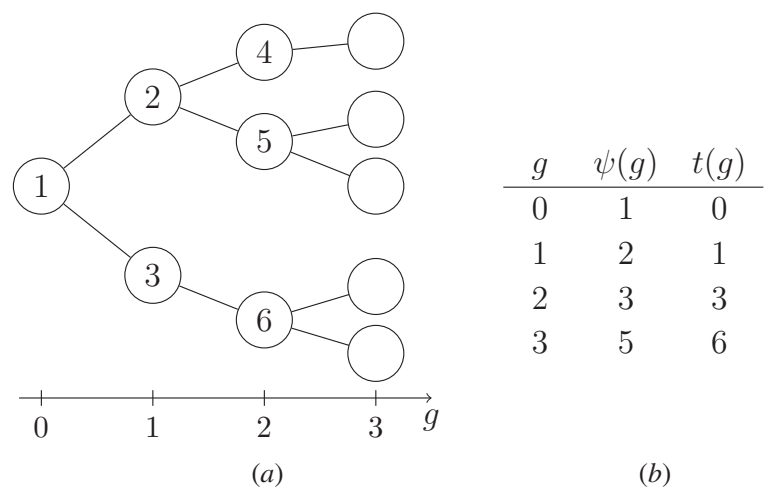

Figure 1. Example of a BP evolving over three generations. The last generation has not yet been updated. The last individual which produced any offspring is the one labelled $t=6$. (a) BP as a tree. (b) Mapping of generational time $g$ and individual time $t$ via the generation size $\psi(g)=\phi(t(g))$.

theorem [4, theorem 3.4.6], which states that continuous local martingales in one dimension can be expressed as time changed Brownian motions, with the new time being the quadratic variation of the process. For the purposes of this paper, we can state this result as follows: we can find a new Brownian motion $\tilde{W}(g)$, such that the stochastic integral in (the integrated version of (2)) can be written

$$
\int_{0}^{t} \sqrt{\sigma(\psi(g))} \mathrm{d} W(g)=\tilde{W}\left(\int_{0}^{t} \sigma(\psi(g)) \mathrm{d} g\right) .
$$

By construction, this change of the clock works only in one dimension.

Just as with the Lamperti transformation (3), this transformation can also be performed at the level of the Fokker-Planck equation. Even though the Dambis-Dubins-Schwarz theorem is a standard result in stochastic analysis that has been used for the theoretical analysis of SDEs in one dimension (and also for the proof of homogenization theorems with error estimates [12]) it has not been used, to our knowledge, in the calculation of statistical quantities of interest for SDEs with multiplicative noise, in particular when boundary conditions have to be taken into account. More precisely, the connection between the change of the clock at the level of the SDE and the study of branching processes (BPs) is not known. It is the goal of this paper to study precisely this problem and apply our insight to the analysis of the random neighbour contact process.

The following section motivates the mapping and (for illustrative purposes) exemplifies it using a continuum formulation of the branching process. In that case essentially all results are known in closed form. In section 3 we proceed to apply the mapping to the random neighbour contact process, which will be turned into an Ornstein-Uhlenbeck process with absorbing wall. Section 4 contains a discussion of the results.

\section{Branching process}

The mapping employed in the following between a random walk (RW) and a Watson-Galton $\mathrm{BP}$, is very well established in the literature [13-16]. We adopt the language of a (family) tree, such as the one shown in figure 1. The BP can be studied at two different time scales, the slow generational time $g$ (as the one indicated on the axis in figure $1(a)$ ) and the fast individual time $t$ which corresponds to the labelling of the nodes shown in figure 1(a). Using 
the mapping as described below, the labelling within a generation and thus the fast time scale remain somewhat arbitrary, which is irrelevant for the argument.

A branching tree can be considered as having 'grown' generation by generation by allowing each individual within a generation to reproduce. In figure $1(a)$ this is indicated by the labels of the nodes. If $\psi(g)$ is the number of individuals in generation $g$, the fast (microscopic) time scale $t$ may be defined as

$$
t(g)=\sum_{g^{\prime}=0}^{g-1} \psi(g),
$$

namely the total number of reproduction attempts that occurred up to (but excluding) generation $g$, the slow (macroscopic) time scale. With obvious generalizations in mind, the following discussion is restricted to a BP with two reproduction attempts for each node, implemented by two independent Bernoulli trials with probability $p$, i.e. two offspring are produced with probability $p^{2}$, a single one with $2 p(1-p)$ and none with $(1-p)^{2}$. In this setup, the evolution of the BP can be guided by a RW of $\phi(t)$ which may change by at most 1 at two consecutive times. The population size $\psi(g+1)$ of generation $g+1$ is then determined by $\phi(t(g+1))$ which has taken $\psi(g)$ time steps since $t(g)$, so that $\phi(t(g))=\psi(g)$. No further evolution can take place once $\psi(g)$, or, for that matter, $\phi(t)$, has vanished. In other words, the RWs to be considered are those along an absorbing wall.

Each time step $t$ of the random walker corresponds to a reproduction attempt of an individual in the previous generation, as indicated by the labelling in figure 1 $(a)$, which is not unique, yet can be interpreted as a particular realization of the partial reproduction attempt of a generation. This picture therefore affords a bijection between RW and BP.

\subsection{Continuum formulation}

To make further progress, the mapping is re-formulated in the continuum on the basis of the definition

$$
t(g)=\int_{0}^{g} \mathrm{~d} g^{\prime} \psi\left(g^{\prime}\right)
$$

and $\phi(t(g))=\psi(g)$. Since $t(0)=0$ the initial conditions used below will be $\psi_{0}=\phi_{0}$. To make equation (7) a bijection, $\psi(g)$ and thus $\phi(t)$ may not vanish. Equation (7) is then easily inverted,

$$
g(t)=\int_{0}^{t} \mathrm{~d} t^{\prime} \frac{1}{\phi\left(t^{\prime}\right)} .
$$

If $\phi(t)$ has the equation of motion of a random walker along an absorbing wall with drift $\epsilon$, we have

$$
\frac{\mathrm{d}}{\mathrm{d} t} \phi(t) \equiv \dot{\phi}(t)=\epsilon+\xi(t)
$$

where $\xi(t)$ is a noise with correlator

$$
\left\langle\xi(t) \xi\left(t^{\prime}\right)\right\rangle=2 \Gamma^{2} \delta\left(t-t^{\prime}\right),
$$

with $\langle\cdot\rangle$ denoting the ensemble average. From equation (9) follows

$$
\frac{\mathrm{d}}{\mathrm{d} g} \psi(g)=\frac{\mathrm{d} t}{\mathrm{~d} g} \dot{\phi}(t)=\psi(g)(\epsilon+\xi(t(g)))
$$

because $\frac{\mathrm{d} t}{\mathrm{~d} g}=\psi(g)$ from equation (7). The equation of motion (11) for $\psi(g)$ can be further simplified by introducing the noise

$$
\left\langle\eta(g) \eta\left(g^{\prime}\right)\right\rangle=2 \Gamma^{2} \delta\left(g-g^{\prime}\right)=2 \Gamma^{2} \delta\left(t-t^{\prime}\right) \frac{\mathrm{d} t}{\mathrm{~d} g}
$$


or, equivalently,

$$
\eta(g)=\sqrt{\psi(g)} \xi(t(g))
$$

which results in the final continuum version of the equation of motion of the branching process ${ }^{1}$

$$
\frac{\mathrm{d}}{\mathrm{d} g} \psi(g)=\psi(g) \epsilon+\sqrt{\psi(g)} \eta(g) .
$$

The term $\sqrt{\psi(g)} \eta(g)$ reflects the fact that the variance of the size of each generation is linear in its previous size (where the term 'previous' reminds us of the Itō interpretation of equation (14)).

The derivation of the SDE (14) involving multiplicative noise from the one involving additive noise, equation (9), is invertible, i.e. (7) implies (9) given (14).

Mathematically, the construction above on the level of a Langevin equation is at best handwaving: Because the random variable $\psi(g)$ enters into the definition (7) of time $t$, time itself becomes a random variable. Worse, the definition of the noise via its correlator in equation (12) involves the random variable $\mathrm{d} t / \mathrm{d} g$.

At the level of a Fokker-Planck equation, the transform amounts to a change of variables, yet unlike, say, [17, appendix A], one of the time variable, involving the entire history of the random variable, equation $(7)$.

\subsection{Generalization of the mapping}

The mapping performed above can be generalized as follows: a Langevin equation of the form

$$
\frac{\mathrm{d}}{\mathrm{d} g} \psi(g)=\mu(\psi(g))+\sigma(\psi(g)) \eta(g)
$$

with white noise $\eta(g)$ as defined in equation (12), is equivalent to

$$
\frac{\mathrm{d}}{\mathrm{d} t} \phi(t)=\frac{\mu(\phi(t))}{\sigma^{2}(\phi(t))}+\xi(t)
$$

for $\phi(t(g))=\psi(g)$ along an absorbing wall,

$$
t(g)=\int_{0}^{g} \mathrm{~d} g^{\prime} \sigma^{2}\left(\psi\left(g^{\prime}\right)\right)
$$

and white noise $\xi(t)$ as defined in equation (10). However, the distribution of $\psi(g)$ at fixed $g$ does not equal the distribution of $\phi(t)$ at fixed $t$, because the map $t(g)$ involves the history of $\psi$. Some observables, however, do not change under the mapping and can be used to identify a transition, which is illustrated in section 2.4.

\subsection{Fokker-Planck equations and solutions}

To understand the meaning and the consequences of the mapping introduced above, we obtain solutions for the Fokker-Planck equations of both, the RW along an absorbing wall with additive noise (9) and the BP with multiplicative noise (14). Because for the latter the case $\epsilon=0$ can be recovered from the general solution with $\epsilon \neq 0$ only in the form of a limit, and because $\epsilon<0$ is qualitatively different from $\epsilon>0$, these three cases will be discussed separately.

1 The continuum version of the BP retains some crucial features of the discrete counterpart, so that, for example, asymptotic population sizes in the latter can be calculated on the basis of the former using suitable effective parameters $\Gamma^{2}$ and $\epsilon$. This carries through even to the total population sizes, $\Psi$, calculated below. 
It is a textbook exercise to find the Fokker-Planck equation for the random walker along an absorbing wall, equation (9), which is

$$
\partial_{t} \mathcal{P}^{(\phi)}\left(\phi, t ; \phi_{0} ; \Gamma^{2}, \epsilon\right)=\Gamma^{2} \partial_{\phi}^{2} \mathcal{P}^{(\phi)}-\epsilon \partial_{\phi} \mathcal{P}^{(\phi)},
$$

with $\mathcal{P}^{(\phi)}\left(\phi, t ; \phi_{0} ; \Gamma^{2}, \epsilon\right)$ the probability of finding the walker at $\phi$ at time $t$, given it started from $\phi_{0}$ at $t=0$,

$$
\lim _{t \rightarrow 0} \mathcal{P}^{(\phi)}\left(\phi, t ; \phi_{0} ; \Gamma^{2}, \epsilon\right)=\delta\left(\phi-\phi_{0}\right)
$$

and given the amplitude of the noise $\Gamma^{2}$, equation (10), and the drift $\epsilon$. The absorbing wall implies a Dirichlet boundary condition

$$
\mathcal{P}^{(\phi)}\left(0, t ; \phi_{0} ; \Gamma^{2}, \epsilon\right)=0
$$

and $\phi_{0}>0$. The solution

$$
\mathcal{P}^{(\phi)}\left(\phi, t ; \phi_{0} ; \Gamma^{2}, \epsilon\right)=\frac{1}{\sqrt{4 \pi \Gamma^{2} t}}\left(\mathrm{e}^{-\frac{\left(\phi-\epsilon t-\phi_{0}\right)^{2}}{4 \Gamma^{2} t}}-\mathrm{e}^{-\frac{\left(\phi-\epsilon t+\phi_{0}\right)^{2}}{4 \Gamma^{2} t}} \mathrm{e}^{-\frac{\phi_{0} \epsilon}{\Gamma^{2}}}\right)
$$

is easily obtained using a mirror charge to find the solution of equation (18) whose $\epsilon$ is gauged away using a function $\gamma$ (say, $\left.\gamma(\phi, t)=\exp \left\{\frac{\epsilon}{2 \Gamma^{2}}(x-\epsilon t / 2)\right\}\right)$ and writing $\mathcal{P}^{(\phi)}=\gamma \tilde{\mathcal{P}}^{(\phi)}$, or other methods, e.g. [18].

The Fokker-Planck equation of the continuous BP, equation (14), is

$$
\partial_{g} \mathcal{P}^{(\psi)}\left(\psi, g ; \psi_{0} ; \Gamma^{2}, \epsilon\right)=\Gamma^{2} \partial_{\psi}^{2}\left(\psi \mathcal{P}^{(\psi)}\right)-\epsilon \partial_{\psi}\left(\psi \mathcal{P}^{(\psi)}\right)
$$

for $\psi>0$, with initial condition, $\psi_{0}=\psi(0)=\phi_{0}$,

$$
\lim _{g \rightarrow 0} \mathcal{P}^{(\psi)}\left(\psi, g ; \psi_{0} ; \Gamma^{2}, \epsilon\right)=\delta\left(\psi-\psi_{0}\right)
$$

Its solution is

$$
\begin{aligned}
\mathcal{P}^{(\psi)}\left(\psi, g ; \psi_{0} ;\right. & \left.\Gamma^{2}, \epsilon\right)=\sqrt{\frac{\psi_{0}}{\psi}} \frac{\epsilon \exp (-\epsilon g / 2)}{\Gamma^{2}(1-\exp (-\epsilon g))} I_{1}\left(\frac{2 \epsilon \sqrt{\psi \psi_{0} \exp (-\epsilon g)}}{\Gamma^{2}(1-\exp (-\epsilon g))}\right) \\
& \times \exp \left(-\frac{\psi \exp (-\epsilon \psi)+\psi_{0}}{\Gamma^{2}(1-\exp (-\epsilon g))}\right)
\end{aligned}
$$

where $I_{1}$ denotes the modified Bessel function of the first kind. The prefactor $\epsilon /(1-\exp (-\epsilon g))$ has two important properties. Firstly, for $g>0$ it is positive for all non-vanishing $\epsilon$. Secondly, taking the limit

$$
\lim _{\epsilon \rightarrow 0} \frac{\epsilon}{1-\exp (-\epsilon g)}=\frac{1}{g}
$$

recovers the solution of equation (22) for $\epsilon=0$,

$$
\mathcal{P}^{(\psi)}\left(\psi, g ; \psi_{0} ; \Gamma^{2}, \epsilon=0\right)=\sqrt{\frac{\psi_{0}}{\psi}} \frac{1}{\Gamma^{2} g} I_{1}\left(\frac{2 \sqrt{\psi \psi_{0}}}{\Gamma^{2} \psi}\right) \mathrm{e}^{-\frac{\psi+\psi_{0}}{\Gamma^{2} g}} .
$$

In fact, the latter solution is found in tables [19-21]. From that, equation (24) is obtained after a sequence of transforms. Firstly, introducing $\mathcal{P}^{(\psi)}\left(\psi, g ; \psi_{0} ; \Gamma^{2}, \epsilon\right)=$ : $R\left(\psi \exp (-\epsilon \psi), \psi \Gamma^{2}\right) / \psi$ simplifies equation $(22)$ to $\dot{R}(x, \tilde{s})=x \exp \left(-\epsilon \tilde{s} / \Gamma^{2}\right) R^{\prime \prime}(x, \tilde{s})$. In order to absorb the prefactor, we introduce $R(x, \tilde{s})=-\left(\Gamma^{2} / \epsilon\right) S\left(x, \exp \left(-\epsilon \tilde{s} / \Gamma^{2}\right)\right)$, so that $\dot{S}(x, b)=-\left(\Gamma^{2} / \epsilon\right) x S^{\prime \prime}(x, b)$. If $\mathcal{P}^{(\psi)}\left(\psi, g ; \psi_{0} ; \Gamma^{2}, 0\right)$ solves equation (22) with $\epsilon=0$, then $S(x, b)=A x \mathcal{P}^{(\psi)}\left(x, b-1 ; \psi_{0},-\Gamma^{2} / \epsilon\right)$ with the same boundary and initial condition as above. The initial condition applies at $b=1$, the value of $\exp \left(-\epsilon \tilde{s} / \Gamma^{2}\right)$ at $\tilde{s}=0$. Some algebra then recovers the full solution equation (24). 


\subsection{Comparison of the branching process and the random walker picture}

In the following we compare a range of observables between the different processes or, rather, their description. In fact, equations (21) and (24) are only two different perspectives on the same process, with the advantage that one (the former, with additive noise) is much easier to obtain and analyse than the other.

In the Langevin equations (14) and (9), $\psi$ and $\phi$ respectively do not recover from having vanished. In the following the limits

$$
\begin{aligned}
& \lim _{t \rightarrow \infty} \mathcal{N}^{(\phi)}\left(t ; \phi_{0} ; \Gamma^{2}, \epsilon\right)=: \mathcal{N}_{0}^{(\phi)}\left(\phi_{0} ; \Gamma^{2}, \epsilon\right) \\
& \lim _{g \rightarrow \infty} \mathcal{N}^{(\psi)}\left(g ; \psi_{0} ; \Gamma^{2}, \epsilon\right)=: \mathcal{N}_{0}^{(\psi)}\left(\psi_{0} ; \Gamma^{2}, \epsilon\right)
\end{aligned}
$$

of the integrals

$$
\begin{aligned}
& \mathcal{N}^{(\phi)}\left(t ; \phi_{0} ; \Gamma^{2}, \epsilon\right):=\int_{0}^{\infty} \mathrm{d} \phi \mathcal{P}^{(\phi)}\left(\phi, t ; \phi_{0} ; \Gamma^{2}, \epsilon\right) \\
& \mathcal{N}^{(\psi)}\left(g ; \psi_{0} ; \Gamma^{2}, \epsilon\right):=\int_{0}^{\infty} \mathrm{d} \psi \mathcal{P}^{(\psi)}\left(\psi, g ; \psi_{0} ; \Gamma^{2}, \epsilon\right)
\end{aligned}
$$

are referred to as the asymptotic survival probabilities. Inspecting equation (7) shows that indefinite survival in the BP may not map to indefinite survival of a random walker if $\psi\left(g^{\prime}\right)$ vanishes fast enough. In turn, if $\phi\left(t^{\prime}\right)$ diverges quickly enough, $g(t)$ in equation (8) might remain finite in the limit $t \rightarrow \infty$. Yet, equation (21) indicates that the distribution of $\phi(t)$ is centred around $\phi_{0}-\epsilon t$ and events beyond that scale are exponentially suppressed. Indefinite survival of a random walker thus results in (typically logarithmic) divergence of $g(t)$ in $t$.

We conclude that survival of a random walker corresponds to survival of a corresponding BP and, likewise, early death (at finite $g$ ) of a BP corresponds to an early death of a random walker (at finite $t$ ). One may therefore expect that a transition from asymptotic death to asymptotic survival in one system corresponds to a corresponding transition in the other system.

Integrating equations (21) and (24) according to equation (28a) gives

$\mathcal{N}^{(\phi)}\left(t ; \phi_{0} ; \Gamma^{2}, \epsilon\right)=\frac{1}{2}\left(1-\mathrm{e}^{-\frac{\epsilon \phi_{0}}{\Gamma^{2}}}\left(1+\mathcal{E}\left(\frac{\epsilon t-\phi_{0}}{2 \sqrt{\Gamma^{2} t}}\right)\right)+\mathcal{E}\left(\frac{\epsilon t+\phi_{0}}{2 \sqrt{\Gamma^{2} t}}\right)\right)$

and

$\mathcal{N}^{(\psi)}\left(g ; \psi_{0} ; \Gamma^{2}, \epsilon\right)= \begin{cases}1-\exp \left(-\frac{-\psi_{0} \epsilon}{\Gamma^{2}(1-\exp (-\epsilon g))}\right) & \text { for } \epsilon \neq 0 \\ 1-\exp \left(-\frac{-\psi_{0}}{\Gamma^{2} g}\right) & \text { for } \epsilon=0\end{cases}$

which is again continuous in $\epsilon$. Taking, however, the long time limits gives

$$
\begin{gathered}
\mathcal{N}_{0}^{(\phi)}\left(\phi_{0} ; \Gamma^{2}, \epsilon\right)= \begin{cases}1-\exp \left(-\frac{-\phi_{0} \epsilon}{\Gamma^{2}}\right) & \text { for } \epsilon>0 \\
0 & \text { for } \epsilon \leqslant 0\end{cases} \\
\mathcal{N}_{0}^{(\psi)}\left(\psi_{0} ; \Gamma^{2}, \epsilon\right)= \begin{cases}1-\exp \left(-\frac{-\psi_{0} \epsilon}{\Gamma^{2}}\right) & \text { for } \epsilon>0 \\
0 & \text { for } \epsilon \leqslant 0\end{cases} \\
7
\end{gathered}
$$


which is, as expected, in agreement, because $\psi_{0}=\phi_{0}$. A discrepancy is however expected in the leading order behaviour in large $t$ and $g$ respectively, for $\epsilon \leqslant 0$, which is, for the random walker

$\mathcal{N}^{(\phi)}\left(t, \phi_{0} ; \Gamma^{2}, \epsilon\right) \sim \begin{cases}1-\mathrm{e}^{-\frac{-\phi_{0} \epsilon}{\Gamma^{2}}} & \text { for } \epsilon>0 \\ \frac{\phi_{0}}{\sqrt{\pi \Gamma^{2} t}} & \text { for } \epsilon=0 \\ \frac{2 \phi_{0}}{\left(\epsilon t+\phi_{0}\right)\left(\epsilon t-\phi_{0}\right)} \sqrt{\frac{\Gamma^{2} t}{\pi} e^{-\frac{\left(\epsilon t+\phi_{0}\right)^{2}}{4 \Gamma^{2} t}}} & \text { for } \epsilon<0\end{cases}$

and for the BP

$$
\mathcal{N}^{(\psi)}\left(g, \psi_{0} ; \Gamma^{2}, \epsilon\right) \sim \begin{cases}1-\mathrm{e}^{-\frac{\psi_{0} \epsilon}{\Gamma^{2}}} & \text { for } \epsilon>0 \\ \frac{\psi_{0}}{\Gamma^{2} g} & \text { for } \epsilon=0 \\ \frac{\psi_{0}|\epsilon|}{\Gamma^{2}} \mathrm{e}^{\epsilon g} & \text { for } \epsilon<0\end{cases}
$$

The case $\epsilon=0$, obviously the critical point, deserves special attention. It is well known that the survival probability in a fair BP, $\epsilon=0$, is inverse in the number of generations [13]. Since the expected population size remains unchanged in the fair case, $\langle\psi\rangle\left(g, \psi_{0} ; \Gamma^{2}, \epsilon=0\right)=\psi_{0}$, as discussed below, the expected population size conditional to survival is $\Gamma^{2} g$ according to equation $(33 b)$. On that basis, equation (7) suggests $t(g) \approx(1 / 2) \Gamma^{2} g^{2}$ which produces $\phi_{0} /\left(\Gamma^{2} g \sqrt{\pi / 2}\right)$ in $(32 b)$, out by a factor $1 / \sqrt{\pi / 2}$ compared to the asymptote for the BP at $\epsilon=0$, equation $(33 b)$.

Other observables worth comparing are moments conditional to survival, as they are stationary. The moments

$$
\left\langle\psi^{n}\right\rangle=\int_{0}^{\infty} \mathrm{d} \psi \psi^{n} \mathcal{P}^{(\psi)}\left(\psi, g ; \psi_{0} ; \Gamma^{2}, \epsilon\right)
$$

of equation (24) can be calculated very easily using the identity

$$
\int_{0}^{\infty} \mathrm{d} x I_{1}(x) \mathrm{e}^{-\gamma x^{2}}=\mathrm{e}^{1 /(4 \gamma)}-1
$$

and differentiating with respect to $\gamma$ which gives

$\int_{0}^{\infty} \mathrm{d} \psi \psi^{-1 / 2} I_{1}(\sqrt{\psi} \alpha) \mathrm{e}^{\beta \psi} \psi^{n}=\left.2 \alpha^{-(2 n+1)}\left(-\frac{\mathrm{d}}{\mathrm{d} \gamma}\right)^{n}\right|_{\gamma=-\beta / \alpha^{2}}\left(\mathrm{e}^{1 /(4 \gamma)}-1\right)$.

We find, for example,

$$
\begin{aligned}
\left\langle\psi^{0}\right\rangle & =1-\mathrm{e}^{-\frac{\psi_{0} \epsilon}{\Gamma^{2}(1-\exp (-\epsilon g))}} \\
\left\langle\psi^{1}\right\rangle & =\psi_{0} \mathrm{e}^{\epsilon g} \\
\left\langle\psi^{2}\right\rangle & =\left\langle\psi^{1}\right\rangle^{2}+2 \psi_{0} \frac{\Gamma^{2}}{\epsilon}\left(\mathrm{e}^{2 \epsilon g}-\mathrm{e}^{\epsilon g}\right),
\end{aligned}
$$

where we use the convention $\left\langle\psi^{0}\right\rangle=\mathcal{N}^{(\psi)}\left(g, \psi_{0} ; \Gamma^{2}, \epsilon\right)$ and similarly for $\phi$. Corresponding expressions for the random walker are extremely messy (as can be seen in equation (29)), so we state only the first moment (the zeroth moment is stated in equation (29) and its expansion in equation (32b)), dropping lower order terms in $t$ (1.o.t.):

$$
\left\langle\phi^{1}\right\rangle= \begin{cases}\epsilon t\left(1-\mathrm{e}^{-\frac{\epsilon \phi_{0}}{\Gamma^{2}}}\right)+\text { 1.o.t. } & \text { for } \epsilon>0 \\ \phi_{0} & \text { for } \epsilon=0 \\ \frac{8 \phi_{0}|\epsilon| \Gamma^{2} t^{2}}{\left(\epsilon t+\phi_{0}\right)^{2}\left(\epsilon t-\phi_{0}\right)^{2}} \sqrt{\frac{\Gamma^{2} t}{\pi}} \mathrm{e}^{-\frac{\left(\epsilon t+\phi_{0}\right)^{2}}{4 \Gamma^{2} t}}+1 . \text { o.t. } & \text { for } \epsilon<0\end{cases}
$$


Normalizing the moments with the respective survival probability gives the moments conditional to survival, $\left\langle\psi^{n}\right\rangle_{s}=\left\langle\psi^{n}\right\rangle / \mathcal{N}^{(\psi)}$ and similar for $\left\langle\phi^{n}\right\rangle_{s}$. For $\epsilon>0$ the normalization is the same in both cases, equation (31a), so a comparison of the conditional moments comes in fact down to comparison of the unconditional moments. For $\psi \approx \psi_{0} \exp (\epsilon g)$ the mapped time is $t(g)=\psi_{0}(\exp (\epsilon g)-1) / \epsilon$ which in equation $(38 b)$ gives $\langle\phi\rangle=$ $\psi_{0}(\exp (\epsilon g)-1)\left(1-\exp \left(-\frac{\epsilon \phi_{0}}{\Gamma^{2}}\right)\right)$, not quite matching equation $(37 b)$.

For $\epsilon=0$ the unconditional moments are identical, equation (37b) (at $\epsilon=0$ ) and equation (38b), so the comparison of the conditional moments is merely a comparison of the normalizations $(32 b)$ and $(33 b)$, which has been addressed above.

The case $\epsilon<0$ gives a conditional first moment of $\lim _{t \rightarrow \infty}\left\langle\phi^{1}\right\rangle_{s}=\lim _{t \rightarrow \infty}\left\langle\phi^{1}\right\rangle /\left\langle\phi^{0}\right\rangle=$ $4 \Gamma^{2} /|\epsilon|$ while the BP gives $\lim _{g \rightarrow \infty}\left\langle\psi^{1}\right\rangle_{s}=\lim _{g \rightarrow \infty}\left\langle\psi^{1}\right\rangle /\left\langle\psi^{0}\right\rangle=\Gamma^{2} /|\epsilon|$.

At first one may expect time independent quantities to be equal in both setups. However, as they remain subject to their dynamics, survivors in one system (say the BP) may generally be much closer to death than survivors in the other (say the random walkers), as they continue to linger close to extinction.

One observable that can be recovered in the random walker mapping in exact form is the total population size ${ }^{2}$ in the BP

$$
\Psi=\int_{0}^{\infty} \mathrm{d} g^{\prime} \psi\left(g^{\prime}\right)
$$

whose expectation is finite if $\epsilon<0$ and corresponds, according to the mapping (7), exactly to the time a random walker hits the absorbing wall. This is easily confirmed for the first moment, since

$$
\langle\Psi\rangle=\int_{0}^{\infty} \mathrm{d} g^{\prime}\left\langle\psi\left(g^{\prime}\right)\right\rangle=\frac{\psi_{0}}{|\epsilon|}
$$

for $\epsilon<0$ and because the probability density of walkers to hit the wall at $t$ is $\left(-\frac{\partial}{\partial t}\right) \mathcal{N}^{(\phi)}\left(t ; \phi_{0} ; \Gamma^{2}, \epsilon\right)$, in the random walker picture

$$
\left\langle\Psi^{n}\right\rangle=\int_{0}^{\infty} \mathrm{d} t t^{n}\left(-\frac{\partial}{\partial t}\right) \mathcal{N}^{(\phi)}\left(t ; \phi_{0} ; \Gamma^{2}, \epsilon\right)
$$

One thus easily finds for $\epsilon<0$

$$
\begin{aligned}
\left\langle\Psi^{1}\right\rangle & =\frac{\phi_{0}}{|\epsilon|} \\
\left\langle\Psi^{2}\right\rangle & =\langle\Psi\rangle^{2}+\frac{2 \Gamma^{2} \phi_{0}}{|\epsilon|^{3}} \\
\left\langle\Psi^{3}\right\rangle & =\frac{\phi_{0}}{\left|\epsilon^{5}\right|}\left(12 \Gamma^{4}+6 \Gamma^{2}|\epsilon| \phi_{0}+\epsilon^{2} \phi_{0}^{2}\right) \\
& =\langle\Psi\rangle^{3}+3\left(\left\langle\Psi^{2}\right\rangle-\langle\Psi\rangle^{2}\right)\langle\Psi\rangle+\frac{12 \Gamma^{4} \phi_{0}}{\left|\epsilon^{5}\right|}
\end{aligned}
$$

obtained straightforwardly in the random walker picture. In contrast, deriving higher moments of $\Psi$ in the BP picture is quite cumbersome, as they require higher correlation functions, for example

$$
\left\langle\Psi^{2}\right\rangle=\int_{0}^{\infty} \mathrm{d} g^{\prime} \int_{0}^{\infty} \mathrm{d} g^{\prime \prime}\left\langle\psi\left(g^{\prime}\right) \psi\left(g^{\prime \prime}\right)\right\rangle .
$$

\footnotetext{
2 In self-organized criticality the total population size is in fact the time-integrated activity or, equivalently, the avalanche size [22].
} 
In the presence of the 'kernel' $\mathcal{P}^{(\psi)}$, equation (24), this correlation function is determined via

$$
\left\langle\psi\left(g^{\prime}\right) \psi\left(g^{\prime \prime}\right)\right\rangle=\int_{0}^{\infty} \mathrm{d} \psi^{\prime} \psi^{\prime} \mathcal{P}^{(\psi)}\left(\psi^{\prime}, g^{\prime} ; \psi_{0} ; \Gamma^{2}, \epsilon\right) \int_{0}^{\infty} \mathrm{d} \psi^{\prime \prime} \psi^{\prime \prime} \mathcal{P}^{(\psi)}\left(\psi^{\prime \prime}, g^{\prime \prime}-g^{\prime} ; \psi^{\prime} ; \Gamma^{2}, \epsilon\right)
$$

assuming $g^{\prime \prime}>g^{\prime}$. The resulting expressions can be evaluated using the moments calculated above, equations (37a)-(37c). As expected, they exactly coincide with equations $(42 a)-(42 c)$.

\section{Contact process}

We will now use the mapping illustrated above to characterize the stochastic equation of motion of the random neighbour contact process.

The contact process [1] is a simple lattice model, for example of the spatio-temporal evolution of an immobile plant species spreading on a substrate. For definiteness set up on a square lattice, sites are either occupied or empty. Occupied sites become empty with Poissonian extinction rate $e$ and attempt to occupy with an offspring each of their neighbouring sites with the same rate $c / q$, where $q$ is the number of neighbours, in case of nearest neighbour interaction, $q$ being the coordination number. Such an attempt is successful, resulting in occupation of the empty site, only when the targeted site was empty prior to the attempt. The interaction is thus due to excluded volume, as colonization can occur only at empty sites. Sites become occupied with Poissonian colonization rate $k c / q$, where $k$ is the number of occupied nearest neighbours. Extensions to higher and lower dimensions are obvious. Rescaling the time by $e$ determines the single parameter controlling the dynamics as $\lambda=c / e$. It turns out [23] that in the thermodynamic limit a finite population density of occupied sites $\psi$ is sustained for all $\lambda$ greater than some $\lambda_{c}$, displaying all features of a second order phase transition [24].

In fact, these features can already be seen in a mean field theory, where the rise in occupation density is given by the competition of extinction and global colonization as a function of time $g$

$$
\frac{\mathrm{d}}{\mathrm{d} g} \psi=\lambda(1-\psi) \psi-\psi
$$

which has a non-trivial stationary state $\psi=1-1 / \lambda>0$ for $\lambda>1$, i.e. $\lambda_{c}=1$. In fact, to leading order $\psi \propto\left(\lambda-\lambda_{c}\right)^{-1}$ for $\lambda \gtrsim \lambda_{c}$ and $\psi=0$ otherwise. To go beyond mean field theory, two additional ingredients are needed, namely spatial interaction and noise. The former is implemented by smoothing out the occupation by introducing a diffusion term. The latter accounts for the stochastic nature of the process. Similar to the BP analysed in section 2, the variance of fluctuations should be linear in the local occupation. The full Langevin equation of motion that is usually analysed as the contact process [1] reads $^{3}$

$\dot{\psi}(\mathbf{x}, g)=\lambda(1-\psi(\mathbf{x}, g)) \psi(\mathbf{x}, g)-\psi(\mathbf{x}, g)+D \nabla^{2} \psi+\sqrt{\psi(\mathbf{x}, t)} \eta(\mathbf{x}, g)$

where the noise has vanishing mean, is Gaussian, white and has correlator

$$
\left\langle\eta(\mathbf{x}, g) \eta\left(\mathbf{x}^{\prime}, g^{\prime}\right)\right\rangle=2 \Gamma^{2} \delta\left(g-g^{\prime}\right) \delta\left(\mathbf{x}-\mathbf{x}^{\prime}\right) .
$$

This equation has been analysed extensively using field theoretic methods [25-27], in particular perturbation theory. Above the upper critical dimension $d>d_{c}=4[28,29]$ spatial

3 The absence of an explicit carrying capacity and extinction rate spoils the usual dimensional independence of $\psi$. 


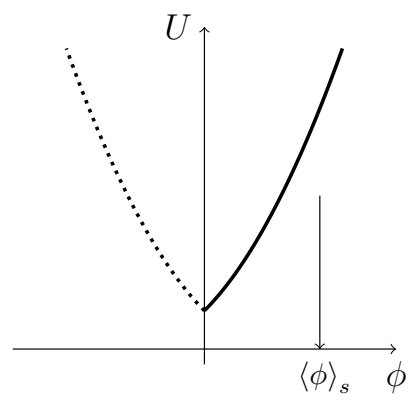

(a) $\lambda=1 / 2$

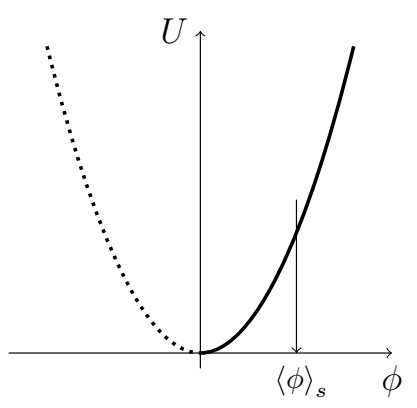

(b) $\lambda=1$

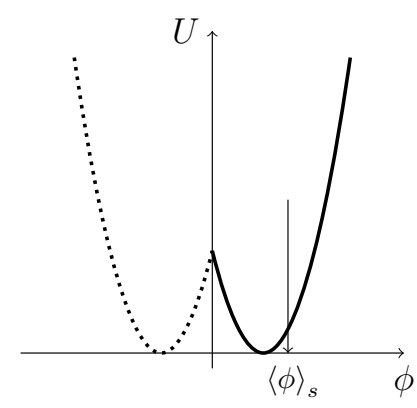

(c) $\lambda=3$

Figure 2. The potential, equation (50), for three different values of $\lambda$. Mirror charges are subject to the dotted potential on the left. The arrow from above marks the position of the expected average position $\phi$ conditioned on survival.

variation of $\psi$ becomes irrelevant ${ }^{4}$, i.e. the diffusion term can be dropped, resulting in the random neighbour contact process,

$$
\frac{\mathrm{d}}{\mathrm{d} g} \psi(g)=\lambda(1-\psi(g)) \psi(g)-\psi(g)+\sqrt{\psi(g)} \eta(g),
$$

which is the equation we will analyse in the following. The aim is to characterize (48) by mapping it onto a linear Langevin equation with additive noise. Firstly this sheds light on the meaning of the random neighbour model and its relation to the original, spatial version. This will also provide a well-founded interpretation of the finite size scaling behaviour of the random neighbour contact process as one would implement it numerically. Secondly, a number of authors have used equations of the form above to model various natural phenomena [30-34], such as the stochastic logistic equation, and we expect their work to benefit directly from our analytical approach.

\subsection{Mapping the random neighbour CP to an Ornstein-Uhlenbeck process}

Using the mapping introduced in section 2.2 the original equation of motion of the random neighbour contact process equation (48) can be mapped to

$$
\dot{\phi}(t)=-\lambda \phi(t)+(\lambda-1)+\xi(t)
$$

with an absorbing wall at $\phi=0$ and $t(g)$ again given by equation (7).

Was it not for the absorbing wall, the probability density $\mathcal{P}_{0}(\phi)$ in the stationary state could be read off instantly as the deterministic part of (49) can be written as

$$
-\lambda \phi(t)+(\lambda-1)=-\frac{\mathrm{d} U}{\mathrm{~d} \phi} \quad \text { where } \quad U(\phi)=\frac{1}{2} \lambda\left(\phi-\frac{\lambda-1}{\lambda}\right)^{2}
$$

and therefore $\mathcal{P}_{0}(\phi) \propto \exp \left(-U(\phi) / \Gamma^{2}\right)$. The parameter $(\lambda-1) / \lambda$ translates the minimum of the harmonic potential horizontally, while $\lambda$ itself modifies its curvature, see figure 2 . The dotted potential shown there is experienced by the mirror charges placed in the system to meet the Dirichlet boundary condition, producing a double-parabola potential. The cusp of the potential at $\phi=0$ for $\lambda \neq 1$ is indicative of the technical difficulties ahead.

4 Irrelevance is to be understood in the technical, field-theoretical sense. The cartoon normally drawn is that of spatial correlations (on sufficiently large scales) no longer entering, when the dimension is so high that the process rarely returns to a site. 


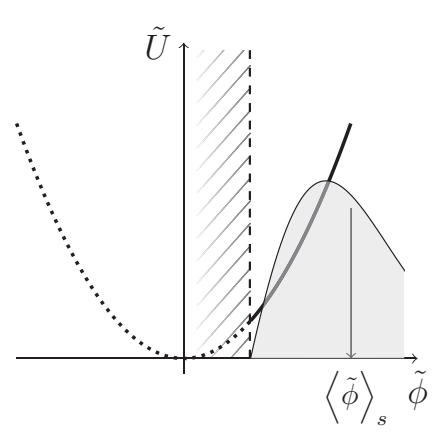

(a) $\lambda=1 / 2, \tilde{a}=1$

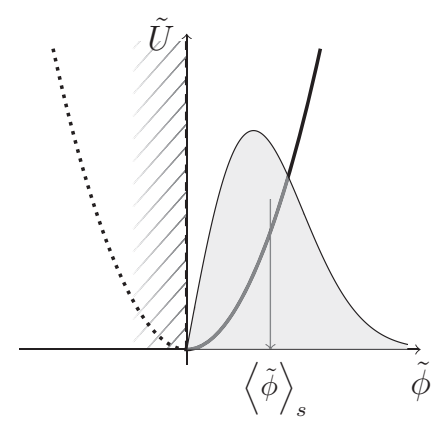

(b) $\lambda=1, \tilde{a}=0$

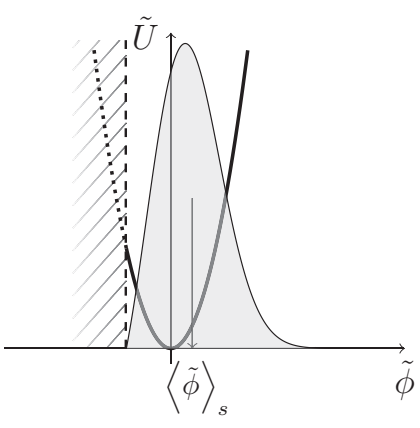

(c) $\lambda=3, \tilde{a}=-2 / 3$

Figure 3. The potential $\tilde{U}(\tilde{\phi})=U(\phi)=(1 / 2) \lambda \tilde{\phi}^{2}$ (as in equation (50)) of the OrnsteinUhlenbeck process equation (51) after the shift by $\tilde{a}=-1+1 / \lambda$, equation (52). The absorbing wall is indicated by the dashed line and the hatched region, which is not accessible for the walker. The grey, shaded areas are the asymptotic conditional probability densities equation (64). The arrow from above marks the position of the expected average position $\tilde{\phi}$ conditioned on survival, see figure 2 .

One may expect a phase transition due to the competition of two scales: The distance between the absorbing wall and the minimum of the potential, $(\lambda-1) / \lambda$, and the strength of the noise relative to the steepness of the potential, $\Gamma / \sqrt{\lambda}$, which may or may not drive the particles into the wall. Because the ratio of the two lengths is dimensionless, it is possible that the transition occurs at a non-trivial value of $\lambda$ giving rise to non-trivial exponents.

To ease notation, the origin is now moved so that the minimum of the potential is at $\tilde{\phi}=0$, resulting in the Langevin equation analysed in the following

$$
\dot{\tilde{\phi}}(t)=-\lambda \tilde{\phi}(t)+\xi(t)
$$

where

$$
\tilde{\phi}(t)=\phi(t)+\tilde{a} \quad \text { with } \quad \tilde{a}=\frac{1}{\lambda}-1
$$

the latter being the position of the absorbing wall in a shifted potential, illustrated in figure 3 . The Langevin equation (51) is an Ornstein-Uhlenbeck process [35] with an absorbing wall [36].

\subsection{The Fokker-Planck equation}

In the following, we will determine the 'solution' of equation (51), which is expected to be much more easily obtained than that of the original process (46). The Fokker-Planck equation reads [37]

$$
\frac{\partial}{\partial t} \mathcal{P}(\tilde{\phi} ; t)=\lambda \frac{\partial}{\partial \tilde{\phi}}(\tilde{\phi} \mathcal{P}(\tilde{\phi} ; t))+\Gamma^{2} \frac{\partial^{2}}{\partial \tilde{\phi}^{2}} \mathcal{P}(\tilde{\phi} ; t)
$$

with boundary condition $\mathcal{P}(\tilde{a} ; t)=0$ and initial condition $\lim _{t \rightarrow 0} \mathcal{P}(\tilde{\phi} ; t)=\delta\left(\tilde{\phi}-\tilde{\phi}_{0}\right)$. It is obvious to attempt to write its solution in terms of eigenfunctions $y_{n}(x)$ with $x=\tilde{\phi} \sqrt{\lambda} / \Gamma$ and eigenvalues $\mu_{n}$, say

$$
\mathcal{P}(\tilde{\phi} ; t)=\sum_{n} \mathrm{e}^{-\mu_{n} \lambda t} \mathrm{e}^{-\frac{\lambda \tilde{\phi}^{2}}{2 \Gamma^{2}}} y_{n}(x)
$$


where $y_{n}$ fulfils the eigenvalues equation

$$
y_{n}^{\prime \prime}-x y_{n}^{\prime}=-\mu_{n} y_{n},
$$

with $y_{n}(a)=0$. The factor $\exp \left(-\lambda \tilde{\phi}^{2} /\left(2 \Gamma^{2}\right)\right)$ in (54) appears quite naturally; without it, the eigenvalues $\mu_{n}$ were negative and each term in the series divergent. Equation (55) is in fact the eigenvalue problem of the Kolmogorov backward operator (generator) [9] of (53) and for $\mu_{n} \in \mathbb{N}(55)$ is the Hermite equation.

However, Hermite polynomials do not generally solve (55), because they do not generally fulfil $y_{n}(\tilde{a})=0$, except when $\tilde{a}=0$ (i.e. the potential in figure $2(a)$ without a cusp), where $y_{n}(x)=H_{m}(x)$ for $n=0,1,2, \ldots$ and $m=2 n+1$, so that $\mu_{n}=m$. This solution in odd polynomials hints at the interpretation of the problem in terms of a mirror charge trick alluded to earlier.

The Kolmogorov backward equation (55) is also a Sturm-Liouville eigenvalue problem and multiplication by the weight function $\mathrm{e}^{-\frac{x^{2}}{2}}$ converts it to standard Sturm-Liouville form

$$
\left(\mathrm{e}^{-\frac{x^{2}}{2}} y_{n}^{\prime}(x)\right)^{\prime}+\mu_{n} \mathrm{e}^{-\frac{x^{2}}{2}} y_{n}(x)=0 \text {. }
$$

A Sturm-Liouville eigenvalue problem has a set of eigenvalues corresponding to a complete set of orthogonal eigenfunctions that are square integrable with respect to the weight function [38]. This equation is a singular Sturm-Liouville problem because it is defined on an infinite interval and therefore an extra condition is needed such that $\sqrt{\mathrm{e}^{-\frac{x^{2}}{2}}} y_{n}(x)$ tends to zero as $|x| \longrightarrow \infty$, to ensure square integrability of $y_{n}(x)$. If this condition holds then a complete set of solutions can be found.

Solutions of (55) can be constructed in terms of a series by studying the recurrence relation of its coefficients. A more efficient route is the use of special functions, such as confluent hypergeometric functions also known as Kummer functions [39]. These are solutions of the differential equation [40] $x \frac{\mathrm{d}^{2} y}{\mathrm{~d} x^{2}}+(\beta-x) \frac{\mathrm{d} y}{\mathrm{~d} x}-\alpha y=0$ where $y(x)=\mathrm{M}(\alpha ; \beta ; x)$ or $y(x)=$ $\mathrm{U}(\alpha ; \beta ; x)$ or any linear combination thereof. A solution of the form $y=\mathrm{U}\left(-\mu_{n} / 2 ; 1 / 2 ; x^{2} / 2\right)$ solves equation (55) and satisfies the Dirichlet boundary condition at $\tilde{a}$ for suitably chosen $\mu_{n}$. It turns out that of the two independent solutions, only $U$ has the right asymptotic behaviour in large $x$ to guarantee square integrability. Unfortunately, $\mathrm{U}\left(-\mu_{n} / 2 ; 1 / 2 ; x^{2} / 2\right)$ does not solve the equation for $\tilde{a}<0$ because it has a singularity at zero and can therefore not constitute a complete system of eigenfunctions of the Sturm-Liouville problem.

Another differential equation to consider is the parabolic cylinder function equation

$$
\frac{\mathrm{d}^{2} y}{\mathrm{~d} x^{2}}+\left(v+\frac{1}{2}-\frac{x^{2}}{4}\right) y=0
$$

The solutions of this equation [41] are the parabolic cylinder functions $y(x)=D_{v}(x)$, which also solve equation (55) when of the form $y=\mathrm{e}^{\frac{x^{2}}{4}} D_{v}(x)$, specifically $D_{v}(x)=$ $\exp \left(-x^{2} / 4\right) H_{v}(x)$ for integer $v$. For suitable $\mu_{n}$ the parabolic cylinder functions satisfy the boundary condition at $\tilde{a}$, namely $D_{\mu_{n}}(\tilde{a} \sqrt{\lambda} / \Gamma)=0$, and are analytic along the whole real line [42]. The solution of (53) with an absorbing wall at $\tilde{a}$ is thus

$$
\mathcal{P}(\tilde{\phi} ; t)=\mathrm{e}^{-\frac{\lambda\left(\tilde{\phi}^{2}-\tilde{\phi}_{0}^{2}\right)}{4 \Gamma^{2}}} \frac{\sqrt{\lambda}}{\Gamma} \sum_{n=1}^{\infty} h_{n}^{-1} \mathrm{e}^{-\mu_{n} \lambda t} D_{\mu_{n}}\left(\frac{\sqrt{\lambda}}{\Gamma} \tilde{\phi}\right) D_{\mu_{n}}\left(\frac{\sqrt{\lambda}}{\Gamma} \tilde{\phi}_{0}\right)
$$

where $h_{n}^{-1}$ is a normalization constant,

$$
\int_{a \sqrt{\lambda} / \Gamma}^{\infty} \mathrm{d} x D_{\mu_{n}}(x) D_{\mu_{m}}(x)=h_{n} \delta_{n m}
$$

which holds for any pair $\mu_{n}, \mu_{m}$ such that $D_{\mu_{n, m}}(\tilde{a} \sqrt{\lambda} / \Gamma)=0$. Completeness of $D_{\mu_{n}}(x)$ guarantees the initial condition $\lim _{t \rightarrow 0} \mathcal{P}(\tilde{\phi} ; t)=\delta\left(\tilde{\phi}-\tilde{\phi}_{0}\right)$. 


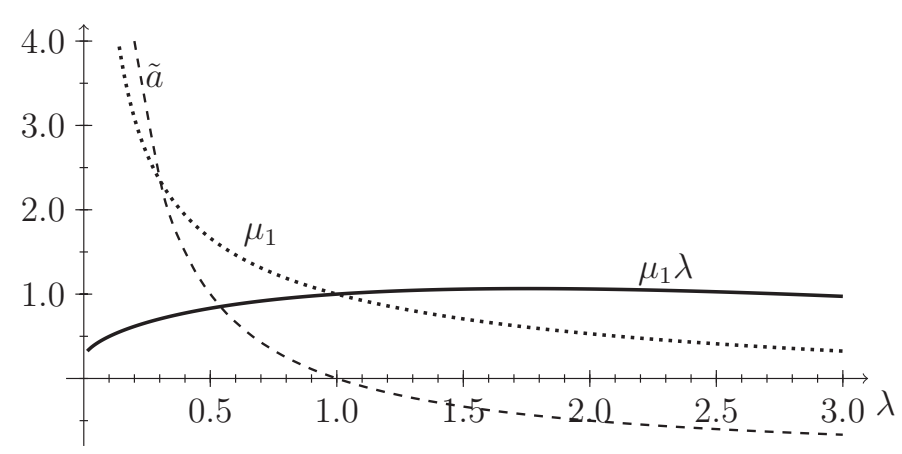

Figure 4. The smallest eigenvalue $\mu_{1}$ (dotted),i.e. the smallest root $\mu$ of $D_{\mu}(\tilde{a} \sqrt{\lambda} / \Gamma)=$ 0 , shown here for $\Gamma=1$ as a function of $\lambda$. The asymptotic death rate of the process, $\mu_{1} \lambda$ (full line), does not signal a phase transition at any finite value of $\lambda$. The position of the absorbing wall, $\tilde{a}=1 / \lambda-1$, is shown as a dashed line.

\subsection{Observables}

In the following the process (51) is characterized with respect to the parameter $\lambda$ with the aim to identify and characterize a phase transition. As above, the observables we are interested in are the survival probability, conditional moments and time-integrated activity. By construction, only the latter can be calculated exactly in the mapping to the Ornstein-Uhlenbeck process.

Based on the solution (58) all observables mentioned above are easily accessible. Asymptotically, the probability density is dominated by the smallest eigenvalue $\mu_{1}$,

$$
\lim _{t \rightarrow \infty} \mathcal{P}(\tilde{\phi} ; t) \mathrm{e}^{\mu_{1} \lambda t}=\frac{\sqrt{\lambda}}{\Gamma} h_{1}^{-1} \mathrm{e}^{-\frac{\lambda\left(\tilde{\phi}^{2}-\tilde{\phi}_{0}^{2}\right)}{4 \Gamma^{2}}} D_{\mu_{1}}\left(\frac{\sqrt{\lambda}}{\Gamma} \tilde{\phi}\right) D_{\mu_{1}}\left(\frac{\sqrt{\lambda}}{\Gamma} \tilde{\phi}_{0}\right)
$$

so that the (relative) death rate is immediately identified as $\mu_{1} \lambda$, which is shown in figure 4 . The rate with which the system empties asymptotically, $\mu_{1} \lambda$, is positive for all positive $\lambda$. Moreover, the spectrum is always discrete. In summary, (sudden) onset of asymptotic survival, as seen in the BP above, is not displayed by this Ornstein-Uhlenbeck system, although this is precisely what is expected in a contact process. This apparent clash is resolved below.

For the following calculations a number of identities are useful, such as the recurrence relations of the parabolic cylinder functions [41],

$\frac{\mathrm{d}}{\mathrm{d} x}\left(\mathrm{e}^{x^{2} / 4} D_{v}(x)\right)=v \mathrm{e}^{x^{2} / 4} D_{\nu-1}(x)$ and $\frac{\mathrm{d}}{\mathrm{d} x}\left(\mathrm{e}^{-x^{2} / 4} D_{v}(x)\right)=-\mathrm{e}^{-x^{2} / 4} D_{v+1}(x)$

so that

$$
D_{v+1}(x)-x D_{v}(x)+v D_{v-1}(x)=0 .
$$

The survival probability, $\mathcal{N}^{(\tilde{\phi})}(t)=\int_{\tilde{a}}^{\infty} \mathrm{d} \tilde{\phi} \mathcal{P}(\tilde{\phi} ; t)$, therefore has asymptote

$$
\mathcal{N}^{(\tilde{\phi})}\left(t, \tilde{\phi}_{0} ; \Gamma^{2}, \epsilon\right) \sim \mathrm{e}^{-\mu_{1} \lambda t} h_{1}^{-1} \mathrm{e}^{-\frac{\lambda\left(\tilde{a}^{2}-\tilde{\phi}_{0}^{2}\right)}{4 \Gamma^{2}}} D_{\mu_{1}-1}\left(\frac{\sqrt{\lambda}}{\Gamma} \tilde{a}\right) D_{\mu_{1}}\left(\frac{\sqrt{\lambda}}{\Gamma} \tilde{\phi}_{0}\right) .
$$

While $\mathcal{N}^{(\tilde{\phi})}$ vanishes in large $t$ for all finite $\lambda$, the asymptotic probability density conditional to survival (see equation (60)),

$$
\lim _{t \rightarrow \infty} \frac{\mathcal{P}(\tilde{\phi} ; t)}{\mathcal{N}^{(\tilde{\phi})}(t)}=\frac{\sqrt{\lambda}}{\Gamma} \mathrm{e}^{-\frac{\lambda\left(\tilde{\phi}^{2}-\tilde{a}^{2}\right)}{4 \Gamma^{2}}} \frac{D_{\mu_{1}}\left(\frac{\sqrt{\lambda} \tilde{\phi}}{\Gamma}\right)}{D_{\mu_{1}-1}\left(\frac{\sqrt{\lambda} \tilde{a}}{\Gamma}\right)},
$$

as shown in figure 3, is stationary, in contrast to the BP discussed above. As expected, the wall is effectively repelling, as walkers close to it are absorbed more readily than those staying 
away from it, or, conversely, if a walker survives, then because it stays well away from the wall. Because $\psi$ cannot run off, but, rather, is contained within a parabolic potential, $t(g)$, (7), does not necessarily diverge with $g$, i.e. vanishing asymptotic survival probability in the random walker picture does not imply the same in the original picture (48).

With (61) and (62) asymptotic moments conditional to survival are found as

$$
\lim _{t \rightarrow \infty}\langle\tilde{\phi}\rangle_{s}=\frac{\Gamma}{\sqrt{\lambda}} \frac{D_{\mu_{1}-2}\left(\frac{\sqrt{\lambda}}{\Gamma} \tilde{a}\right)}{D_{\mu_{1}-1}\left(\frac{\sqrt{\lambda}}{\Gamma} \tilde{a}\right)}+\tilde{a}=\frac{\mu_{1}}{\mu_{1}-1} \tilde{a},
$$

and

$$
\begin{aligned}
\lim _{t \rightarrow \infty}\left\langle\tilde{\phi}^{2}\right\rangle_{s}-\langle\tilde{\phi}\rangle_{s}^{2} & =\frac{\Gamma^{2}}{\lambda}\left(\frac{2 D_{\mu_{1}-3}\left(\frac{\sqrt{\lambda}}{\Gamma} \tilde{a}\right)}{D_{\mu_{1}-1}\left(\frac{\sqrt{\lambda}}{\Gamma} \tilde{a}\right)}-\frac{D_{\mu_{1}-2}^{2}\left(\frac{\sqrt{\lambda}}{\Gamma} \tilde{a}\right)}{D_{\mu_{1}-1}^{2}\left(\frac{\sqrt{\lambda}}{\Gamma} \tilde{a}\right)}\right) \\
& =\frac{\tilde{a}^{2} \mu_{1}}{\left(\mu_{1}-1\right)^{2}\left(\mu_{1}-2\right)}-\frac{2 \Gamma^{2}}{\lambda\left(\mu_{1}-2\right)} .
\end{aligned}
$$

Because the asymptotic conditional distribution $\mathcal{P}(\tilde{\phi} ; t) / \mathcal{N}^{(\tilde{\phi})}(t)$ is stationary, one might expect the conditional moments to be identical in the mapped and in the original process. To interpret the results for the contact process correctly, it is crucial to undo the shift applied in equation (52) as that affects the mapping equation (8). The conditional un-shifted position of the random walker, $\lim _{t \rightarrow \infty}\langle\phi\rangle_{s}=\tilde{a} /\left(\mu_{1}-1\right)$, must be strictly positive by construction, suggesting that $\mu_{1}-1 \propto \tilde{a}$ around $\lambda=1$, as $\tilde{a}$ vanishes.

The special case $\lambda=1$ can be solved explicitly using Hermite polynomials, producing

$$
\lim _{t \rightarrow \infty}\langle\tilde{\phi}\rangle_{s}=\lim _{t \rightarrow \infty}\langle\phi\rangle_{s}=\sqrt{\frac{\pi}{2} \Gamma^{2}} \text { for } \lambda=1
$$

which implies $\mu_{1}=1-\sqrt{2 /\left(\pi \Gamma^{2}\right)}(\lambda-1)$ to leading order in $\lambda$ about 1 and via (66)

$$
\lim _{t \rightarrow \infty}\left\langle\tilde{\phi}^{2}\right\rangle_{s}-\langle\tilde{\phi}\rangle_{s}^{2}=\lim _{t \rightarrow \infty}\left\langle\phi^{2}\right\rangle_{s}-\langle\phi\rangle_{s}^{2}=\left(2-\frac{\pi}{2}\right) \Gamma^{2} \text { for } \lambda=1 .
$$

For very small $0<\lambda \ll 1$ the potential becomes increasingly flat while the wall $\tilde{a}$ is moving further and further to the right. At the wall the potential has slope $\tilde{U}^{\prime}(\tilde{a})=1-\lambda$, while its curvature approaches 0 . As $\mu_{1}$ diverges like $1 /\left(4 \Gamma^{2} \lambda\right)$ [43] with vanishing $\lambda,\langle\tilde{\phi}\rangle_{s}$ diverges with $\tilde{a}$ like $1 / \lambda$ (the arrow in figure 3(a) moving further to the right), while $\langle\phi\rangle_{s}$ converges to $4 \Gamma^{2}$, the conditional relative distance to the wall as shown in figure $2(a)$.

Not unexpectedly, for very large $\lambda$ the wall has no noticeable effect, as $\tilde{a}$ approaches -1 and the potential $\tilde{U}(\tilde{\phi})=(1 / 2) \lambda \tilde{\phi}^{2}$ becomes increasingly sharply peaked

$$
\lim _{t \rightarrow \infty}\left\langle\tilde{\phi}^{2}\right\rangle_{s}-\langle\tilde{\phi}\rangle_{s}^{2} \simeq \frac{\Gamma^{2}}{\lambda}
$$

as if the walker were in a potential without absorbing wall, at stationarity distributed like $\exp \left(-\tilde{U}(\tilde{\phi}) / \Gamma^{2}\right)$. Consequently, $\langle\tilde{\phi}\rangle_{s}$ vanishes asymptotically (figure $3(c)$ ). Given equation (65), its asymptotic behaviour is that of $\mu_{1}$, [43]

$$
\mu_{1} \simeq \sqrt{\frac{\lambda}{2 \pi \Gamma^{2}}} \mathrm{e}^{-\frac{\lambda}{2 \Gamma^{2}}}
$$

which is reminiscent of the Kramers rate of escape over a cusp-shaped barrier [44, section VII.E.2]. Finally, the moments of the total activity in the contact process, as defined in equation (41) for the BP, are easily derived in the random walker picture. By the nature of the mapping, this observable is recovered exactly:

$$
\left\langle\Psi^{m}\right\rangle=\mathrm{e}^{-\frac{\lambda\left(\tilde{a}^{2}-\tilde{\phi}_{0}^{2}\right)}{4 \Gamma^{2}}} \frac{\sqrt{\lambda}}{\Gamma} \sum_{n=1}^{\infty} h_{n}^{-1} m !\left(\mu_{n} \lambda\right)^{-m} D_{\mu_{n}-1}\left(\frac{\sqrt{\lambda}}{\Gamma} \tilde{a}\right) D_{\mu_{n}}\left(\frac{\sqrt{\lambda}}{\Gamma} \tilde{\phi}_{0}\right)
$$


The factor $h_{n}^{-1}$ on the right-hand side ensures quick convergence of the sum. There is, in fact, no suggestion that $\left\langle\Psi^{m}\right\rangle$ is not analytic in $\lambda$.

This concludes the calculation of the observables that are easily derived from the random walker picture of the random neighbour contact process. The moments of the total activity, (71), are exact, while the moments of the asymptotic conditional population density, such as (67) and (68), are not necessarily. None of the observables, however, signals a transition, in contrast to the simplified mean field theory (45).

\section{Discussion and conclusion}

Before we discuss the mapping employed above in broader terms, we want to address the question of why the random neighbour contact process, as formulated in equation (48), does not display the phase transition its mean-field theory exhibits.

The analysis above shows that in the random walker picture, fluctuations will eventually drive the particle into the absorbing wall irrespective of its position and the particle's starting point. Correspondingly, in the original random neighbour contact process, the activity eventually ceases with finite rate as long as the absorbing state is accessible. On the other hand, every naïve numerical implementation of the random walker contact process will display the mean field behaviour. Yet, numerical implementations of absorbing state phase transitions suffer from the problem of being necessarily finite [45]. Taking the thermodynamic limit is crucial for the recovery of the transition, because in any finite system the system almost surely falls into an absorbing state (extinction). At closer inspection, the same applies in the present model: In increasingly large systems with volume $N$ the effective noise amplitude vanishes like $\Gamma^{2} \propto 1 / N$, because the occupation density $\psi$ in a large system is less affected by the noise than in small systems. Decreasing $\Gamma$ has the same effect on $\mu_{n}$ as increasing the magnitude of $\tilde{a} \sqrt{\lambda}$, since $D_{\mu_{n}}(\tilde{a} \sqrt{\lambda} / \Gamma)=0$. In the limit of vanishing $\Gamma$ there are thus three cases:

$$
\lim _{\Gamma \rightarrow 0} \mu_{1}=\left\{\begin{array}{lll}
\infty & \text { for } & \tilde{a} \sqrt{\lambda}>0 \\
1 & \text { for } & \tilde{a} \sqrt{\lambda}=0 \\
0 & \text { for } & \tilde{a} \sqrt{\lambda}<0
\end{array}\right.
$$

It is obviously important to take the limit $\Gamma \rightarrow 0$ in equation (58) before considering its asymptotes in large $t$. For $\tilde{a}<0$ the particles are pinched in an infinitely sharp potential and cannot overcome the barrier to the absorbing wall, i.e. $\lim _{t \rightarrow \infty} \lim _{\Gamma \rightarrow 0}\langle\phi\rangle=-\tilde{a}$, or according to equation (65)

$$
\lim _{\Gamma \rightarrow 0}\langle\phi\rangle_{s}=\lim _{\Gamma \rightarrow 0} \frac{1}{\mu_{1}-1} \tilde{a}=-\tilde{a}=1-\frac{1}{\lambda} \quad \text { for } \quad \lambda>1
$$

as $\tilde{a} \sqrt{\lambda}<0$ in equation (72b), reproducing the mean field result stated after equation (45). The wall becomes accessible for $\tilde{a} \leqslant 0$ in which case $\lim _{t \rightarrow \infty}\langle\phi\rangle_{s}=0$. For $\tilde{a} \sqrt{\lambda}>0$ this is in line with equations (72b) and (65). For $\tilde{a}=0$ the special case (67) applies (because $\lambda=1)$ and taking the limit $\Gamma^{2} \rightarrow 0$ there, produces again $\langle\phi\rangle_{s}=0$. The limit $\Gamma \rightarrow 0$ thus recovers the case $\Gamma=0$ which leads to the mean field theory equation (45) that displays the transition. Taking the limit $\Gamma \rightarrow 0$ directly in equation (58) using equation $(72 b)$ is more difficult, because we were unable to identify a suitable asymptotic behaviour of $D_{\mu_{1}}(\tilde{\phi} \sqrt{\lambda} / \Gamma)$ as $\mu_{1}$ approaches 0 and $\Gamma$ vanishes.

In summary, the phase transition disappears provided the amplitude of the noise correlator is finite, because all walkers will eventually reach the absorbing wall, irrespective of the value of $\lambda$. The transition can thus be partly restored by studying finite $t$, as the characteristic time 
to death is given by the time scale $1 / \lambda$ provided by the potential, multiplied by the distance of the wall relative to the width of $\tilde{\phi}$ produced by the noise, $\tilde{a} \sqrt{\lambda} / \Gamma$. As $\Gamma^{2} \propto 1 / N \propto L^{-d}$ in a $d$-dimensional system with linear extent $L$, this suggests a dynamical exponent of $z=d / 2$, which is correct at the upper critical dimension $d=4$ [46].

In the present random neighbour model the rôle of the thermodynamic limit is thus primarily to suppress fluctuations which always drive the system to extinction. Only once the noise has been eliminated can the long-time limit be taken. One may expect that similar effects play a rôle in spatially extended systems.

\subsection{Mapping multiplicative to additive noise}

The scheme introduced in section 2.2 has a very broad range of applications. If used to cancel a multiplicative noise amplitude, it will typically be applied with the intention to reduce the nonlinearity present in the Langevin equation. This is obviously not automatically the case. For example

$$
\frac{\mathrm{d}}{\mathrm{d} g} \psi(g)=\lambda(1-\psi(g)) \psi(g)-\psi(g)+\sqrt{\psi(g)(1-\psi(g))} \eta(g),
$$

which is a more sophisticated version of the random neighbour contact process that includes fluctuations in the number of unoccupied sites, leads to the mapped equation

$$
\dot{\phi}(t)=-\lambda+\frac{1}{1-\phi(t)}+\xi(t)
$$

somewhat reminiscent of the Bessel process, now equipped with an absorbing wall.

In general, the scheme allows the relation of a range of different Langevin equations and processes, some of which are much easier to analyse than others. A potential disadvantage is that only certain observables are exactly recovered in the mapped process; however, for a large number of processes and mappings this is not of great significance, for example if the primary aim is to identify a phase transition, or if the observables are expected to be sufficiently well approximated in the mapped process.

\section{Acknowledgments}

The authors would like to thank Yang Chen, Michael Gastner, Wolfram Just, Satya Majumdar, Andy Parry, and Hugo Touchette for very valuable discussions. Gunnar Pruessner gratefully acknowledges support from EPSRC through the EPSRC Mathematics Platform grant EP/I019111/1. GAP gratefully acknowledges support from EPSRC through grants $\mathrm{EP} / \mathrm{J} 009636 / 1$ and EP/H034587/1.

\section{References}

[1] Hinrichsen H 2000 Adv. Phys. 49 815-958

[2] Henkel M, Hinrichsen H and Lübeck S 2008 Non-Equilibrium Phase Transitions (Berlin: Springer)

[3] Janssen H K and Stenull O 2012 Phys. Rev. E 85011123

[4] Karatzas I and Shreve S 1991 Brownian Motion and Stochastic Calculus (Graduate Texts in Mathematics vol 113) 2nd edn (New York: Springer)

[5] Wong E and Zakai M 1965 Ann. Math. Statist. 36 1560-4

[6] Kupferman R, Pavliotis G A and Stuart A M 2004 Phys. Rev. E 70036120

[7] Pavliotis G A and Stuart A M 2005 Multiscale Model. Simul. 4 1-35 (electronic)

[8] Pavliotis G 2014 Stochastic processes and applications lecture notes www.ma.imperial.ac.uk/ $\sim$ pavl/stoch_proc_notes.pdf 
[9] Horsthemke W and Lefever R 1984 Noise-induced Transitions: Theory and Applications in Physics, Chemistry, and Biology (Springer Series in Synergetics vol 15) (Berlin: Springer)

[10] Aït-Sahalia Y 2008 Ann. Statist. 36 906-37

[11] Mackey M, Longtin A and Lasota A 1990 J. Statist. Phys. 60 735-51

[12] Hairer M and Pavliotis G 2004 J. Statist. Phys. 117 261-79

[13] Harris T E 1963 The Theory of Branching Processes (Berlin: Springer)

[14] Feller W 1968 An Introduction to Probability Theory and Its Applications 3rd edn vol I (New York: Wiley)

[15] Feller W 1966 An Introduction to Probability Theory and Its Applications vol II (New York: Wiley)

[16] Stapleton M A 2007 Self-organised criticality and non-equilibrium statistical mechanics PhD Thesis Imperial Collage London, University of London (www.matthewstapleton.com/ thesis.pdf)

[17] Baxter G, Blythe R and McKane A 2007 Math. Biosci. 209 124-70

[18] Farkas Z and Fülöp T 2001 J. Phys. A: Math. Gen. 34 3191-8

[19] Wolfram Research Inc. 2011 Mathematica version 8.0.1.0 (Champaign, IL: Wolfram Research)

[20] Research W 2013 Bessel function of the first kind: Differential equations (formula 03.01.13.0005), http://functions.wolfram.com/Bessel-TypeFunctions/BesselJ/13/01/01/0005/ (accessed 16 April 2013)

[21] Gradshteyn I S and Ryzhik I M 2000 Table of Integrals, Series and Products 6th edn (San Diego, CA: Academic) p 690 section 6.615

[22] Pruessner G 2012 Self-Organised Criticality (Cambridge, UK: Cambridge University Press)

[23] Liggett T M 2005 Stochastic Interacting Systems: Contact, Voter and Exclusion Processes (Berlin: Springer)

[24] Fisher M E 1967 Rep. Prog. Phys. 30 615-730

[25] Janssen H K 1981 Z. Phys. B 42 151-4

[26] Grassberger P 1982 Z. Phys. B 47 365-74

[27] Täuber UC 2005 Critical dynamics preprint. Available at www.phys.vt.edu/ tauber/ utaeuber.html (accessed 11 Feb. 2010)

[28] Janssen H, Schaub B and Schmittmann B 1988 Z. Phys. B 71 377-85

[29] Brézin E and Zinn-Justin J 1985 Nucl. Phys. B 257 867-93

[30] Levins R 1969 Proc. Natl Acad. Sci. USA 62 1061-5

[31] Colizza V, Barrat A, Barthélemy M and Vespignani A 2006 Proc. Natl Acad. Sci. USA 103 2015-20

[32] Kaluza P, Kölzsch A, Gastner M T and Blasius B 2010 J. R. Soc. Interface 7 1093-103

[33] Hufnagel L, Brockmann D and Geisel T 2004 Proc. Natl Acad. Sci. USA 101 15124-9

[34] Dean P 1966 Proc. Camb. Phil. Soc. 62 277-86

[35] van Kampen N G 1992 Stochastic Processes in Physics and Chemistry (Amsterdam: Elsevier) (third impression 2001, enlarged and revised)

[36] Finch S 2004 Ornstein-Uhlenbeck Process as of 15 May 2004, available from www.people.fas.harvard.edu/ sfinch/csolve/ou.pdf (accessed 28 Feb. 2013)

[37] Zinn-Justin J 1997 Quantum Field Theory and Critical Phenomena 3rd edn (New York: Oxford University Press)

[38] Al-Gwaiz M A 2008 Sturm-Liouville Theory and its Applications (Berlin: Springer)

[39] Abramowitz M and Stegun I A (ed) 1970 Handbook of Mathematical Functions (New York: Dover)

[40] Slater L J 1960 Confluent Hypergeometric Functions (Cambridge: Cambridge University Press)

[41] Bateman H (United States Office of Naval Research) 1953 Higher Transcendental Functions: Based, in Part, on Notes Left by Harry Bateman vol. 2 (New York: McGraw-Hill)

[42] Whittaker E T and Watson G N 1902 A Course of Modern Analysis: An Introduction to the General Theory of Infinite Processes and of Analytic Functions; with an Account of the Principal Transcendental Functions (Cambridge: Cambridge University Press)

[43] Ben-Naim E and Krapivsky P L 2010 J. Phys. A: Math. Theor. 43495007

[44] Hänggi P, Talkner P and Borkovec M 1990 Rev. Mod. Phys. 62 251-341

[45] Pruessner G 2007 Phys. Rev. E 76061103

[46] Lübeck S 2004 Int. J. Mod. Phys. B 18 3977-4118 